\title{
Loss of Autophagy In Tibial Growth Plate Chondrocytes Causes Increased Chondrocyte Apoptosis In Young Rats With Chronic Renal Insufficiency
}

\author{
Xiao-jian Wang ( $\sim$ wxj990713@163.com) \\ Shanxi Provincial People's Hospital \\ Xiao Lu \\ Shanxi Provincial People's Hospital \\ Song-jia Guo \\ Shanxi Provincial People's Hospital \\ Wei Tian \\ Shanxi Provincial People's Hospital \\ Jian-bo Wu \\ Shanxi Provincial People's Hospital
}

\section{Research Article}

Keywords: Chronic renal insufficiency, Growth plate, Autophagy, Glycogen, Cell apoptosis

Posted Date: October 18th, 2021

DOl: https://doi.org/10.21203/rs.3.rs-861020/v1

License: (c) (i) This work is licensed under a Creative Commons Attribution 4.0 International License. Read Full License 


\section{Abstract}

Background: To observed the effect of autophagy in tibial growth plate chondrocytes on apoptosis in chronic renal insufficiency(CRI) rats.

Method: Male 4-week-old Sprague Dawley(SD) rats were randomly divided into two groups ( $n=20 /$ per group): (1) the normal group was intragastrically administered distilled water; and (2) the CRI group was given a 150 $\mathrm{mg} /(\mathrm{kg} \cdot \mathrm{d})$ adenine suspension. All rats were sacrificed after continuous gavage for 6 weeks. The tibial length and the width of the tibial growth plate were measured using micro-CT. The width of the tibial growth plate was also measured in histological sections at both $4 \mathrm{w}$ and $10 \mathrm{w}$. The level of the autophagy marker Beclin-1 in chondrocytes was measured by immunofluorescence. The level of glycogenin-1, a marker of intracellular glycogen accumulation, was measured by immunohistochemistry in chondrocytes in vivo and in vitro. The apoptosis rate of chondrocytes was measured by the TUNEL method in vivo and in vitro.

Results: The results showed that the length of tibia was shorter and the width of tibia growth plate was narrower in CRF young rats. Autophagy level of chondrocytes in tibial growth plate decreased, and accumulation of glycogen granules in chondrocytes increased significantly. Meanwhile, the apoptosis rate of chondrocytes in tibial growth plate increased.

Conclusion: When CRF occurred in young rats, the autophagy level of chondrocytes in tibial growth plate decreased significantly.As a result, there are not enough autophagic vesicles to swallow glycogen granules in chondrocytes and degrade them into glucose for energy supply, which leads to chondrocyte apoptosis.Autophagy of chondrocytes is at least partly involved in energy metabolism of cells.

\section{Introduction}

Chronic renal insufficiency (CRI) in children refers to persistent, chronic renal dysfunction caused by multiple factors, which results in the retention and deposition of nitrogen-containing metabolites in the body and water, electrolyte and acid-base imbalances and ultimately presents a clinical syndrome with systemic symptoms [1-5]. In addition to the typical clinical symptoms of CRI, children also have a specific manifestation of growth retardation [6-10]. Growth retardation and its profound impact on final adult height have been considered to be a significant obstacle to the full rehabilitation of children with CRI. The literature shows that growth and developmental retardation in children is the result of multiple factors, including heredity, insufficient caloric intake, growth hormone resistance, renal bone disease, etc [11-14].

A typical sign of growth retardation in children is height, and the length of the long bone is the main factor affecting height. The length of the long bone is closely related to the development of the epiphyseal growth plate [15-20]. The literature suggests that the development of long bones in the extremities is mainly accomplished by endochondral ossification [21-25]. The growth plate of human long bone is composed of chondrocytes before puberty.According to the morphological and biological characteristics of chondrocytes, they can be divided into rest zone, proliferation zone and hypertrophy calcification zone from proximal to distal end. Chondrocytes constantly proliferate and differentiate from rest zone to hypertrophic calcified zone, and finally turn into osteoblast, which promotes the elongation of long bones. After puberty, the growth plate is completely calcified, and no chondrocytes differentiate into osteoblast, so the long bone stops growing [26, 27]. In other words, this process relies on the continuous proliferation and differentiation of cartilage chondrocytes in the growth plate of 
the long bone, which eventually become bone cells to lengthen the long bone. Therefore, the proliferation and differentiation of growth plate chondrocytes is a key factor in this process. This process is regulated by many factors. Hence, disturbances in the key regulators of chondrocyte proliferation and differentiation may contribute to impaired linear growth in renal failure. Studies have shown that autophagy in growth plate chondrocytes affects the physiological state of chondrocytes [28-34]. Autophagy is a kind of self-regulatory process in cells in response to harmful external stimuli. Autophagy mainly produces autophagosomes, which combine with intracellular metabolites or redundant organelles to ultimately degrade these components and facilitate cellular adaptation to changes in the external environment and maintain homeostasis of cells. The autophagy process is mainly divided into three steps: initiation and elongation, phagocytosis, and fusion and degradation [35]. At first, autophagic vacuoles are gradually isolated from the rough endoplasmic reticulum under certain conditions. Autophagic vacuoles encapsulate intracytoplasmic substances and then combine with lysosomes to form autophagosomes. The hydrolytic enzymes within lysosomes can degrade the substances within the autophagosomes. Previous studies have showed that autophagy mainly degrades metabolites and damaged organelles. However, with further research, scholars have found that autophagic vacuoles can devour and degrade nutrients in the cytoplasm, including amino acids, fats and glycogen particles [36-39]. Chondrocytes maintain a high level of autophagy because cartilage tissue is a poor nutrient medium. The key proteins related to chondrocyte autophagy are autophagy-related protein-5 (Atg-5), Atg-7, Beclin-1, and Light Chain-3 (LC-3) [40]. Gene knockdown of Atg5 or Atg7 during chondrogenesis causes mild growth retardation accompanied by increased chondrocyte death and a decreased proliferation rate [41]. Studies have also shown that ER stressmediated cell death occurs in growth plate chondrocytes lacking Atg7, which leads to mild growth retardation [42]. It should be noted that because chondrocytes are exposed to poor nutrient conditions, energy intake is essential for their survival. Among these nutrients, glucose is the most direct energy source of chondrocytes. A persistent lack of glucose will lead to chondrocyte apoptosis. Most intracellular glucose is stored in chondrocytes in the form of glycogen, which is degraded into glucose for use by chondrocytes. Recent studies have shown that autophagy is involved in chondrocyte energy metabolism, and autophagosomes can degrade intracellular glycogen to form glucose [43-45].

The aim of our study was to examine changes in chondrocyte autophagy levels, intracellular glycogen accumulation and apoptosis in tibial plateau chondrocytes in the context of CRI to further explore the relationship between autophagy and glycogen metabolism in growth plate chondrocytes.

\section{Materials And Methods}

\section{Animal handing}

This study was approved by the Ethics Committee of Shanxi Provincial People's Hospital (approval number: 2020007). Forty 2-month-old male specific-pathogen free(SPF) grade Sprague Dawley(SD) rats (Animal Experiment Centre of Shanxi Provincial People's Hospital, China) were allowed 2 weeks to acclimate to the housing facility. Environmental conditions included a temperature of $24^{\circ} \mathrm{C} \pm 1^{\circ} \mathrm{C}$, humidity of $53 \% \pm 15 \%$, and a 12:12 $\mathrm{h}$ light:dark cycle with the lights on at 07:00 and off at 19:00. The rats were given access to a sterilized diet and water. The rats were divided into two groups ( $n=20 /$ per group): (1) The normal group was intragastrically administered distilled water; and (2) the CRI group was given a $150 \mathrm{mg} /(\mathrm{kg} \cdot \mathrm{d})$ adenine suspension [46]. All young rats were sacrificed after continuous gavage for 6 weeks. All experiments were performed in accordance with the 
Public Health Service Policy and the Guide for the Care and Use of Laboratory Animals. The study was carried out in compliance with the ARRIVE guidelines.

\section{Relevant Evaluation Indexes In Rats}

The rats were sacrificed 6 weeks after the operation, and urine was collected 24 hours before death. The total protein content of the urine was measured. The concentrations of BUN, Scr, $\mathrm{Ca}, \mathrm{P}, \mathrm{Hb}$ and parathyroid hormone (PTH) were measured by intracardiac blood sampling. Histological sections of the kidney were prepared. Masson staining was performed to observe the glomeruli and renal tubules, including glomerular and tubular status, inflammatory cell infiltration, and renal fibrosis.

Micro-CT was used to measure the lengths of the tibias in the two groups and make comparisons between the two groups. At the same time, micro-CT was used to measure the width of the tibial growth plate. The tibial growth plate width was measured in histological sections, and the ratio was determined (normal group: CRI group).

\section{Immunohistochemical Staining}

Immunofluorescence (IFC) staining was used to detect Beclin-1 (2 $\mu \mathrm{g} / \mathrm{ml}$, ab62557, Abcam, USA).

Immunohistochemistry (IHC) staining was used to detect gycogenin-1 (1:100, sc-271109, Santa Cruz, USA). We compared the two groups of specimens using the ratio of positive cells on immunofluorescence histological sections. Mean Integrated Optical Density (MOD) of chondrocyte was calculated and compared between the two groups on immunofluorescence staining sections of chondrocytes in vitro.

We quantitatively scored the IHC results based on the percentage of positive chondrocytes and the staining intensity as described below. We rated the intensity of staining on a scale of 0 to 3: 0 , negative; 1, weak; 2 , moderate; and 3 , strong. We assigned the following proportional scores: 0 , if $0 \%$ of the chondrocytes showed positive staining; 1 , if up to $1 \%$ of the chondrocytes were stained; 2 , if $2-10 \%$ were stained; 3 , if $11-30 \%$ were stained; 4, if $31-70 \%$ were stained; and 5, if $71-100 \%$ were stained. We then combined the proportion and intensity scores to obtain a total score (range: 0-8) as described previously [47]. The results were assessed by 2 experienced pathologists a blind manner.

\section{Tibial Growth Plate Chondrocytes}

Sterile instruments were used to separate the proximal tibial epiphysis area, and the tissues on both sides of the growth plate were scraped and washed with sterile PBS. Chondrocytes were obtained after the tissue was clipped and digested in collagenase type II. The isolated chondrocytes were cultured in Dulbecco's modified Eagle's medium (DMEM) with $10 \%$ foetal calf serum (FCS) at $37^{\circ} \mathrm{C}$ in a humidified atmosphere of $95 \%$ air and $5 \% \mathrm{CO}_{2}$. Third generation (P3) chondrocytes were used for experiments.

\section{Polymerase chain reaction (PCR)}


Total RNA was extracted from cartilage tissue and chondrocytes by using TRIzol reagent (Thermo Fisher Scientific). The quality and quantity of the total RNA samples were measured using a NanoDrop 2000C spectrophotometer (Thermo Fisher Scientific). RNA with an A260/A280 ratio of 1.8 2.0 was used to analyse mRNA expression. Individual RNA samples ( $1 \mu \mathrm{g}$ per sample) were reverse transcribed into cDNA using the PrimeScript RT master mix kit (Takara, Shiga, Japan) according to the manufacturer's instructions. The relative mRNA expression levels of target genes to the control 18S rRNA were determined by RT-PCR using SYBR Premix Ex TaqTM (Takara) and the specific primers in the IQ5 Multicolour Real-Time PCR detection system (Bio-Rad Laboratories, Hercules, CA, USA). These processes are similar to those performed as previously described[48]. The sequences of the primers were as follows: forward 5'-TGGACGATCAGGCGAAACC-3' and reverse 5'GCTGCGGATGCTCTCAATCT-3' for Beclin-1; and forward 5'-ATGCTGCC ACAAATACCCTTT-3' and reverse 5'GGTAGTGGGCCTTTTATGCCT -3' for glycogenin-1.

\section{Western Blotting}

Growth plate tissue and chondrocyte samples were lysed in RIPA lysis buffer containing PMSF and protease and phosphatase inhibitors (Keygen). Some of the protein was mixed with loading buffer, boiled for 10 min, subjected to SDS-PAGE and transferred to PVDF membranes. After being blocked with $5 \%$ fat-free dry milk in TBST, the blots were probed with primary antibodies. The expression levels of the target proteinsBeclin- 1 ( $1 \mu \mathrm{g} / \mathrm{ml}, \mathrm{ab} 62557$, Abcam, USA) and glycogenin-1 (1:1000, sc-271109, Santa Cruz, USA) relative to the control $\beta$-actin were determined by western blotting. The bound antibodies were detected with horseradish peroxidase (HRP)conjugated secondary antibodies and visualized using an enhanced chemiluminescence reagent. The data were analysed by densitometric analysis with ImageJ software.

\section{Statistical analysis}

SPSS 20.0 (SPSS Inc., Chicago, IL, USA) was used for statistical analysis. All data in this study are expressed as the mean \pm standard deviation (SD). A $P$ value of less than 0.05 was considered statistically significant.

\section{Results}

\section{Rat CRI was successfully modelled}

To investigate the success of the rat CRI model, we examined indicators related to renal function, including the concentrations of BUN, Scr, $\mathrm{Ca}, \mathrm{P}, \mathrm{Hb}$ and PTH in intracardiac blood and total urine proteins for 24 hours. The results showed that the concentrations of BUN and Scr and 24-h total urine proteins in the CRI group were higher than those in the normal group. Blood $\mathrm{Hb}$ in the $\mathrm{CRI}$ group was lower than that in the normal group. There was no significant difference in the blood concentrations of $\mathrm{Ca}, \mathrm{P}$ and PTH between the two groups (Table 1).

The histological kidney sections showed severe tubular dilation and inflammatory cell infiltration in the CRI group, and the degree of fibrosis was severe. No renal tubular dilation or inflammatory cell infiltration were observed in the normal group, and the degree of fibrosis was mild (Figure 1).

Developmental retardation of the tibial growth plate in rats with CRI 
To evaluate the effect of $\mathrm{CRI}$ on the development of the tibia, we measured tibial lengths in rats by using micro-CT, and the results showed that the length of the tibia in the CRI group was shorter than that in the normal group. To further observe the development of the tibial growth plate, we used CT to measure the width of the growth plate, and the results showed that the width of the growth plate in the CRI group was narrower than that of the normal group. Furthermore, the histological measurement of the growth plate width also proved that the width of the tibial growth plate in the CRI group was narrower than that of the normal group (Figure 2).

\section{Changes in growth plate chondrocyte autophagy and glycogen accumulation during CRI in vivo}

To observe the changes in autophagy and glycogen accumulation in tibial growth plate chondrocytes in CRI rats, we used immunohistochemical staining to observe the expression of Becin-1, a marker of autophagy, and glycogenin-1, a marker of intracellular glycogen accumulation. The results showed that compared with those of the normal group, the protein level of Becin-1 decreased and the protein level of glycogenin- 1 increased in the CRI group. Moreover, the TUNEL staining results showed that the apoptosis rate of chondrocytes in the CRI group was significantly increased (Figure 3).

\section{Changes in growth plate chondrocyte autophagy and glycogen accumulation during CRI in vitro}

After isolation and culture of tibial growth plate chondrocytes in vitro, it was found that the chondrocytes in the normal group were round, with short spindles and short synapses. The chondrocytes in the CRI group were long and spindle-shaped, with hypertrophic changes and long synapses. The immunohistochemical staining results showed that the level of Becin-1 was decreased and the level of glycogenin-1 was increased in CRI-group chondrocytes. These results showed decreased chondrocyte autophagy and increased glycogen accumulation during CRI. The TUNEL results also showed that the 72-hour chondrocyte apoptosis rate was significantly higher in the CRI group than the normal group (Figure 4, Figure 5).

\section{Discussion}

We found that tibial growth plate chondrocyte autophagy was at least partially involved in intracellular glycogen degradation and energy supply. Autophagic vacuoles can devour intracellular glycogen granules and then bind to the lysosome to form the autolysosome. Hydrolase in the autolysosome degrades the glycogen granules into glucose for chondrocyte use. When CRI occurs in young rats, the level of autophagy in tibial growth plate chondrocytes decreases, and sufficient autophagic vacuoles do not form in chondrocytes to consume glycogen granules. As a result, there is insufficient glucose in the chondrocytes, which leads to chondrocyte apoptosis due to insufficient energy. The apoptosis of CRI tibial growth plate chondrocytes as a result of decreased autophagy may be related to a failure in glycogen degradation in chondrocytes. The apoptotic rate of growth plate chondrocytes is increased; thus, fewer chondrocytes eventually become osteocytes, resulting in dysplasia of the growth plate and a shortened tibial length.

The length of the long bone depends on the development of the growth plate [21, 22, 24]. Growth plate chondrocytes differentiate from the rest region, the proliferation region, and the hypertrophic calcification region, finally becoming osteocytes to lengthen the long bone. When the number of growth plate chondrocytes decreases, the number of chondrocytes that eventually differentiate into osteocytes also decreases, resulting in shorter bone lengths. Rats aged $4 \mathrm{w}$ were selected to approximate humans aged $4 \llbracket 5$ years, and the rats were killed at $10 \mathrm{w}$, which was equivalent to $9 ₫ 10$ years of human age, and this model simulated the developmental period of 
children. We used classic adenine gavage to establish a rat model of chronic renal insufficiency. At $10 \mathrm{w}$, the renal function-related indexes were tested, and the results showed that the model was successfully established. We found that CRI group rats were very thin, with withered hair, severe hair loss and short body lengths. The length of the tibia was measured in CRI group rats, and it was found that the development of the long bone lagged behind that of normal rats, the growth plate of the proximal tibia was narrowed, and the apoptosis rate of chondrocytes was increased. These results indicated that when CRI occurred, the long bones of the rats became shortened and the growth plates were dysplastic.

Autophagy is an important function that allows chondrocytes to maintain homeostasis. Autophagosomes can be formed to degrade intracellular metabolites, and excess organelles can be degraded when there is external malnutrition to facilitate the energy consumption of chondrocytes. Growth plate cartilage tissue is an avascular structure, suggesting that chondrocytes experience malnutrition and hypoxia. Chondrocytes always maintain a high level of autophagy under normal conditions [29, 30,33]. Traditionally, autophagy maintains cellular homeostasis by degrading metabolites and redundant organelles. However, recent studies have found that autophagy is closely related to the energy metabolism of cells, particularly sugar metabolism. Glycogen is a long chain molecule formed by glucose after the shrinkage reaction and can supply the necessary energy to cells by degrading to form glucose molecules when energy is lacking. Glycogenin-1 plays an important role in the initial stage of glycogen synthesis, and its level in chondrocytes can reflect glycogen accumulation in chondrocytes [49, 50]. The data showed that glycogen levels were high in liver cells and muscle cells $[43,44]$. Studies have shown that there is also a certain amount of glycogen in chondrocytes[51]. Glycogen exists in chondrocytes in two forms: the free form in the cytoplasm and the form consumed in autophagic vacuoles. The free cytoplasmic form of glycogen is phosphorylated and degraded under the action of a catabolic enzyme. In the latter form, autolysosomes are formed after the combination of autophagic vacuoles and lysosomes. Hydrolase in autophagosomes directly degrades glycogen to glucose without phosphorylation. Studies have shown that glucose produced in this manner (without glycogen phosphorylation) is more direct and is an important energy source in chondrocytes. We measured the protein levels of Becin-1, a biomarker of autophagy, in the normal group using immunohistochemistry and found that most chondrocytes expressed this protein, whereas the protein expression of Becin-1 in chondrocytes in the CRI group was significantly decreased. Moreover, glycogen accumulation was measured in chondrocytes. It was found that the level of glycogenin-1, a marker of glycogen accumulation, was significantly increased in the CRI group, whereas the level in the normal group was very low, which indicated that the autophagy level decreased and glycogen accumulation increased in the chondrocytes of $\mathrm{CRI}$ rats. Furthermore, the TUNEL assay was used to detect the apoptosis rate of tibial growth plate chondrocytes in the two groups, which also proved that the apoptosis rate of chondrocytes in the CRI group was higher than that of the normal group in vivo and in vitro.

Why is the suppression of autophagy accompanied by increased apoptosis? Based on these experimental results, we hypothesise that there is a certain level of glycogen storage in chondrocytes, as shown in Figure 5. When autophagy is normal, autophagic vacuoles consume glycogen granules and then combine with lysosomes to form autolysosomes. The hydrolase in autolysosomes degrades glycogen to form glucose for chondrocytes to use. When the autophagy level decreases and the number of autophagic vacuoles decreases, glycogen cannot be consumed and degraded, which leads to glycogen accumulation in chondrocytes; these cells cannot obtain the necessary glucose, which eventually leads to a reduced energy supply and apoptosis.

\section{Conclusions}


In summary,we found that during CRI, the autophagy level of chondrocytes decreases, which leads to a failure in glycogen granule degradation to produce glucose for chondrocyte use and eventually leads to a lack of energy and chondrocyte apoptosis. However, when CRI occurs, there are many factors that cause chondrocyte apoptosis; therefore, we do not know what proportion of apoptosis is caused by decreased chondrocyte autophagy. Chondrocytes exist in a hypoxic environment, and anaerobic glycolysis should be the primary form of nutrient metabolism; therefore, we do not know what proportion of glycogen metabolism is autophagy-mediated. These issues require further research.

\section{Abbreviations}

CRI:Chronic Renal Insufficiency; SD:Sprague Dawley;LC-3: Light Chain-3; Atg-5:Autophagy-related protein-5;SPF: Specific-Pathogen Free; PTH:Pparathyroid hormone; IFC:Immunofluorescence;MOD: Mean Integrated Optical Density; DMEM:Dulbecco's modified Eagle's medium; FCS:Foetal calf serum;P3:Third generation; PCR:Polymerase chain reaction.

\section{Declarations}

Acknowledgements The authors would like to thank Fang Liu for her work on literature search and query. The author also thanks Gang Zheng and Hong Bi for their work on pathology.

Statement of Ethics The authors have no ethical conflicts to disclose. This study was approved by the Ethics Committee of Shanxi Provincial People's Hospital (Approval number: 2020007囚Taiyuan®China)

Disclosure Statement The authors declare that they have no competing financial interests.

Authors' contributions XJ Wang: study design, data acquisition, data analysis, wrote the manuscript. X Lu and SJ Guo: data analysis, discussion of results. W Tian: data acquisition, histochemistry and western blotting analysis. JB Wu: statistical analyses. All authors have approved the submitted manuscript.

Availability of data and materials The datasets used and/or analysed during the current study are available from the corresponding author on reasonable request.

Consent for publication Not applicable.

Funding This work was supported by grants from the China Postdoctoral Science Foundation (2020M680910), Fund Program for the Scientific Activities of Selected Returned Overseas Professionals in Shanxi Province (no.20210019),the Natural Science Foundation of Shanxi Province of China (no. 201701D121161) and the Key Research and Development Projects of Shanxi Province (no. 201803D31160囚201703D321027-4).

Provenance and peer review Not commissioned; externally peer reviewed

Open access This is an open access article distributed in accordance with the Creative Commons Attribution Non Commercia license, which permits others to distribute, remix, adapt, build upon this work non-commercially,and license their derivative works on different terms, provided the original work is properly cited, appropriate credit is given, any changes made indicated, and the use is non-commercial. 


\section{References}

1. Misurac J.Chronic kidney disease in the neonate: etiologies, management, and outcomes.Semin Fetal Neonatal Med. 2017,22:98-103.

2. Akchurin OM.Chronic Kidney Disease and Dietary Measures to Improve Outcomes.Pediatr Clin North Am. 2019,66:247-67.

3. Santoro A, Mandreoli M.Chronic renal disease and risk of cardiovascular morbidity-mortality.Kidney Blood Press Res. 2014,39:142-6.

4. Santoro A, Mandreoli M.Chronic renal disease and risk of cardiovascular morbidity-mortality.Kidney Blood Press Res. 2014,39:142-6.

5. Melo GAA, Rodrigues AB, Firmeza MA, et al.Musical intervention on anxiety and vital parameters of chronic renal patients: a randomized clinical trial.Rev Lat Am Enfermagem. 2018,26:e2978.

6.Furth SL, Alexander DC, Neu AM, et al.Does growth retardation indicate suboptimal clinical care in children with chronic renal disease and those undergoing dialysis? Semin Nephrol. 2001,21:463-9.

7. Fine RN.Etiology and treatment of growth retardation in children with chronic kidney disease and end-stage renal disease: a historical perspective.Pediatr Nephrol. 2010,25:725-32.

8.Lewy JE, Van JJ.Somatomedin and growth retardation in children with chronic renal insufficiency.Kidney Int. $1978,14: 361-4$.

9.Fine RN.Growth hormone treatment of children with chronic renal insufficiency, end-stage renal disease and following renal transplantation-update 1997.J Pediatr Endocrinol Metab. 1997,10:361-70.

10.Rhie YJ, Yoo JH, Choi JH, et al.Long-term safety and effectiveness of growth hormone therapy in Korean children with growth disorders: 5-year results of LG Growth Study.PLoS One. 2019,14:e0216927.

11. Furth SL.Growth and nutrition in children with chronic kidney disease.Adv Chronic Kidney Dis.

2005,12:366-71.

12.Bizzarri C, Lonero A, Delvecchio M, et al.Growth hormone treatment improves final height and nutritional status of children with chronic kidney disease and growth deceleration.J Endocrinol Invest. 2018,41:325-31.

13.Fine RN.Growth hormone and the kidney: the use of recombinant human growth hormone (rhGH) in growthretarded children with chronic renal insufficiency.J Am Soc Nephrol. 1991,1:1136-4.

14.Kuizon BD, Salusky IB.Growth retardation in children with chronic renal failure.J Bone Miner Res. 1999,14:1680-9.

15.Mizuhashi K, Ono W, Matsushita Y, et al.Resting zone of the growth plate houses a unique class of skeletal stem cells.Nature. 2018,563:254-8. 
16.Pazzaglia UE, Reguzzoni M, Casati L, et al.Long bone human anlage longitudinal and circumferential growth in the fetal period and comparison with the growth plate cartilage of the postnatal age. Microsc Res Tech. 2019,82:190-8.

17. Haraguchi R, Kitazawa R, Kohara Y, et al.Recent Insights into Long Bone Development: Central Role of Hedgehog Signaling Pathway in Regulating Growth Plate.Int J Mol Sci. 2019,20:5840.

18. Fuente R, Gil-Pena H, Claramunt-Taberner D, et al.Marked alterations in the structure, dynamics and maturation of growth plate likely explain growth retardation and bone deformities of young Hyp mice. Bone. 2018,116:18795.

19.Wei X, Hu M, Mishina Y, et al.Developmental Regulation of the Growth Plate and Cranial Synchondrosis.J Dent Res. 2016,95:1221-9.

20.Growth plate stress distribution implications during bone development: a simple framework computational approach.Guevara JM, Moncayo MA, Vaca-Gonzalez JJ, et al.Comput Methods Programs Biomed. 2015,118:5968.

21.Hinton RJ, Jing Y, Jing J,et al.Roles of Chondrocytes in Endochondral Bone Formation and Fracture Repair.J Dent Res. 2017,96:23-30.

22.Schlundt C, El Khassawna T, Serra A,et al.Macrophages in bone fracture healing: Their essential role in endochondral ossification.Bone. 2018,106:78-89.

23.Bando Y, Sakashita H, Taira F,et al.Origin and development of septoclasts in endochondral ossification of mice.Histochem Cell Biol. 2018, 149:645-54.

24.Tong W, Tower RJ, Chen C, et al.Periarticular Mesenchymal Progenitors Initiate and Contribute to Secondary Ossification Center Formation During Mouse Long Bone Development.Stem Cells. 2019,37:677-89.

25.Nordberg RC, Wang $\mathrm{H}$, Wu Q,et al.Corin is a key regulator of endochondral ossification and bone development via modulation of vascular endothelial growth factor A expression.J Tissue Eng Regen Med. 2018,12:2277-86.

26. Vortkamp A, Lee K, Lanske B, et al.Regulation of rate of cartilage differentiation by Indian hedgehog and PTHrelated protein.Science. 1996,2;273:613-22.

27.Kindblom JM, Nilsson O, Hurme T, et al.Expression and localization of Indian hedgehog (Ihh) and parathyroid hormone related protein (PTHrP) in the human growth plate during pubertal development.J Endocrinol. 2002 ,174:R1-6.

28. Matsuzaki T, Alvarez-Garcia O, Mokuda S, et al.FoxO transcription factors modulate autophagy and proteoglycan 4 in cartilage homeostasis and osteoarthritis.Sci Transl Med. 2018,10:eaan0746.

29. Kang X, Yang W, Feng D, et al.Cartilage-Specific Autophagy Deficiency Promotes ER Stress and Impairs Chondrogenesis in PERK-ATF4-CHOP-Dependent Manner.J Bone Miner Res. 2017,32:2128-41.

30. Ding J, Jin F, Zhao X, et al.Hemiepiphysiodesis stapling induces ER stress apoptosis and autophagy in rat growth plates.Am J Transl Res. 2019,11:1486-97.

Page $10 / 16$ 
31.Srinivas V, Bohensky J, Shapiro IM.Autophagy: a new phase in the maturation of growth plate chondrocytes is regulated by HIF, mTOR and AMP kinase.Cells Tissues Organs. 2009,189:88-92.

32.Bohensky J, Shapiro IM, Leshinsky S, et al.PIM-2 is an independent regulator of chondrocyte survival and autophagy in the epiphyseal growth plate.J Cell Physiol. 2007,213:246-51.

33.Bohensky J, Leshinsky S, Srinivas V, et al.Chondrocyte autophagy is stimulated by HIF-1 dependent AMPK activation and mTOR suppression.Pediatr Nephrol. 2010,25:633-42.

34.Bohensky J, Terkhorn SP, Freeman TA,et al.Regulation of autophagy in human and murine cartilage: hypoxiainducible factor 2 suppresses chondrocyte autophagy. Arthritis Rheum. 2009,60:1406-15.

35.Srinivas V, Bohensky J, Zahm AM,et al.Autophagy in mineralizing tissues: microenvironmental perspectives.Cell Cycle. 2009,8:391-3.

36.Kuma A, Hatano $M$, Matsui $M$, et al.The role of autophagy during the early neonatal starvation period.Nature.2004,432:1032-6

37.Karsli-Uzunbas G, Guo JY, Price S, et al.Autophagy is required for glucose homeostasis and lung tumor maintenance.Cancer Discov. 2014,4:914-927.

38.Singh R, Kaushik S, Wang Y, et al.Autophagy regulates lipid metabolism.Nature,2009,458:1131-5.

39.Rambold AS, Cohen S, Lippincott-Schwartz J.Fatty acid trafficking in starved cells: regulation by lipid droplet lipolysis, autophagy, and mitochondrial fusion dynamics.Dev Cell,2015,32:678-692. DOI:

10.1016/j.devcel.2015.01.029.

40. Masaaki K, Satoshi W, Masato K, et al.Homeostatic levels of p62 control cytoplasmic inclusion body formation in autophagy-deficient mice.Cell. 2007,131:1149-63.

41. Kabeya Y, Mizushima N, Ueno T, et al.LC3, a mammalian homologue of yeast Apg8p, is localized in autophagosome membranes after processing.EMBO J. 2000,19:5720-8.

42. Yoji H, Hiroko IY, Satoshi W,et al.Loss of autophagy in chondrocytes causes severe growth retardation.Autophagy. 2020,16:501-11.

43. Kotoulas OB, Kalamidas SA, Kondomerkos DJ.Glycogen autophagy in glucose homeostasis. Pathol Res Pract,2006;202(9):631-38.

44.Kondomerkos DJ, Kalamidas SA, Kotoulas OB, et al.Glycogen autophagy in the liver and heart of newborn rats. The effects of glucagon, adrenalin or rapamycin[J].Histol Histopathol,2005,20(3):689-96.

45.Kalamidas SA, Kotoulas OB.Glycogen autophagy in newborn rat hepatocytes. Histol Histopathol, 2000,15(4):1011-18.

46.Huang T, Dong Z.Osthole protects against inflammation in a rat model of chronic kidney failure via suppression of nuclear factor-kB, transforming growth factor- $\beta 1$ and activation of phosphoinositide 3kinase/protein kinase B/nuclear factor (erythroid-derived 2)-like 2 signaling.Mol Med Rep. 2017,16:4915-21. 
47. Liang J, Cao R, Wang X, et al. Mitochondrial PKM2 regulates oxidative stress-induced apoptosis by stabilizing Bcl2. Cell Res. 2017,27:329-51.

48. Wei L SX, Terek R, Chen Q. CD95 induced osteoarthritic chondrocytes apoptosis and necrosis: dependency on p38 mitogen-activated protein kinase. Arthritis Res. Ther 2006;8:R37.

49. Guidotti S, Minguzzi M, Platano D,et al.Glycogen Synthase Kinase-3ß Inhibition Links Mitochondrial Dysfunction, Extracellular Matrix Remodelling and Terminal Differentiation in Chondrocytes.Sci Rep. 2017,7:1-14.

50.Hedberg-Oldfors C, Mensch A, Visuttijai K, et al.Polyglucosan myopathy and functional characterization of a novel GYG1 mutation.Acta Neurol Scand. 2018,137:308-15.

51.Cinque L, Forrester A, Bartolomeo R, et al.FGF signalling regulates bone growth through autophagy.Nature. 2015,528:272-5.

\section{Table}

Table 1 Results of related items in animal models of CRI young rats

\begin{tabular}{llllllll} 
& Blood BUN & Blood Scr & Blood Ca & Blood P & Blood Hb & Blood PTH & $\begin{array}{l}\text { Urine } \\
\text { Protein }\end{array}$ \\
\hline & $\mathrm{mmol} / \mathrm{L}$ & $\mu \mathrm{mol} / \mathrm{L}$ & $\mathrm{mmol} / \mathrm{L}$ & $\mathrm{mmol} / \mathrm{L}$ & $\mathrm{g} / \mathrm{L}$ & $\mathrm{ng} / \mathrm{L}$ & $\mathrm{mg}$ \\
\hline Normal & $4.65 \pm 1.89$ & $21.32 \pm 6.13$ & $2.77 \pm 0.76$ & $3.01 \pm 1.31$ & $126.7 \pm 8.1$ & $134.2 \pm 16.9$ & $32 \pm 5.8$ \\
\hline CRI & $14.32 \pm 5.01 *$ & $45.82 \pm 10.21 *$ & $2.88 \pm 0.74$ & $3.15 \pm 0.88$ & $90.1 \pm 10.32 *$ & $128.3 \pm 17.1$ & $127 \pm 7.9 *$
\end{tabular}

* $p<0.05$ compared with the Normal group.

\section{Figures}

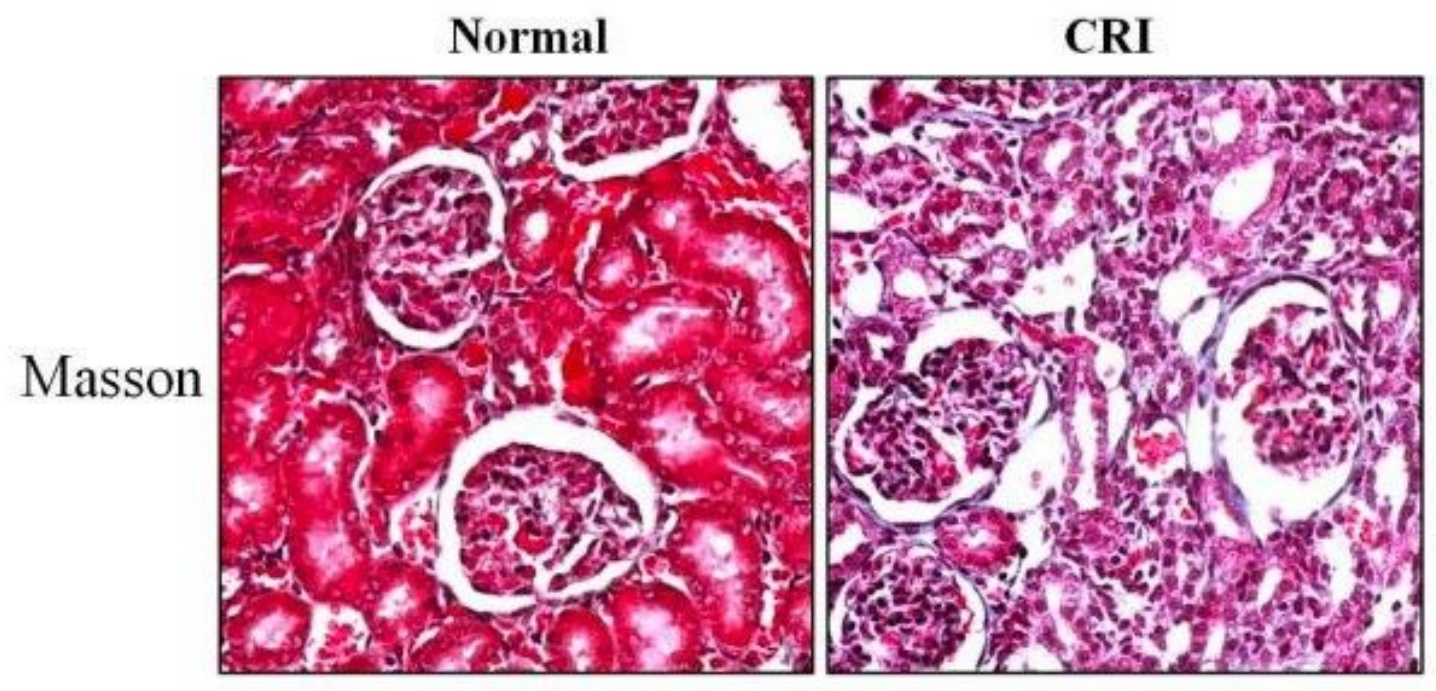


Histological sections of rat kidneys. Histological sections were stained with Masson's trichrome, and in the normal group, there was no dilation of renal tubules and no obvious inflammatory cell infiltration of glomeruli. However, there was obvious dilation of renal tubules and a large number of inflammatory cells infiltrating around the glomeruli in the CRI group $(\times 200)$.
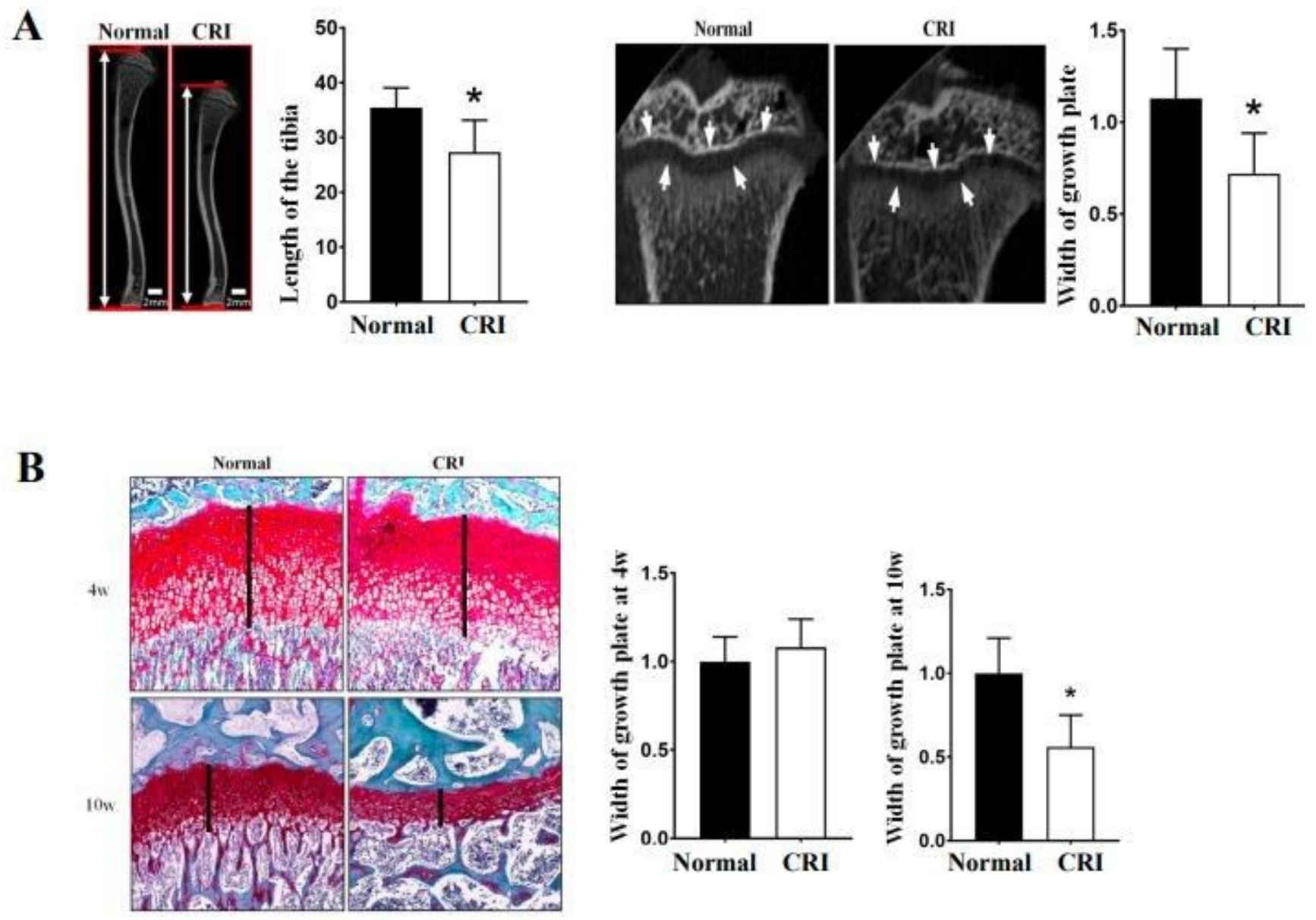

Figure 2

Comparison of tibia length and growth plate width in vitro. (A) Micro-CT measurement results showed that the length of the tibia in the CRI group was shorter than that in the normal group. (B) Micro-CT measurement results showed that the width of the tibial growth plate in the CRI group was shorter than that in the normal group. (C) Histological section measurement results showed that the growth plate width of the CRI group was narrower than that of the normal group at $10 \mathrm{w}(\times 100)$. Bars represent the mean $\pm S E M,{ }^{*} p<0.05$ compared with the normal group. 
A
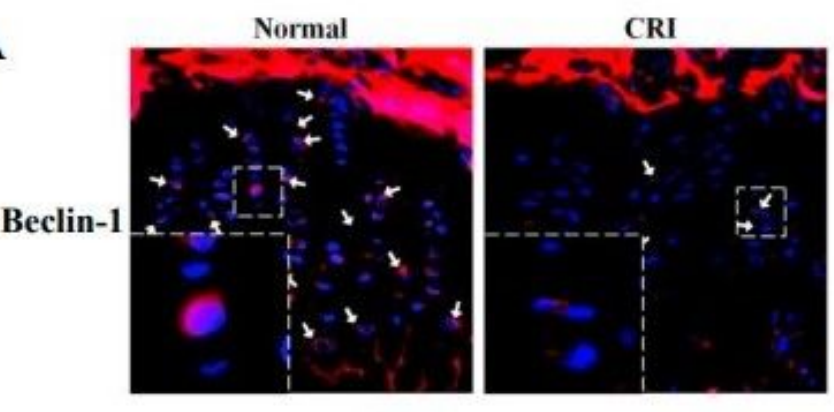

B

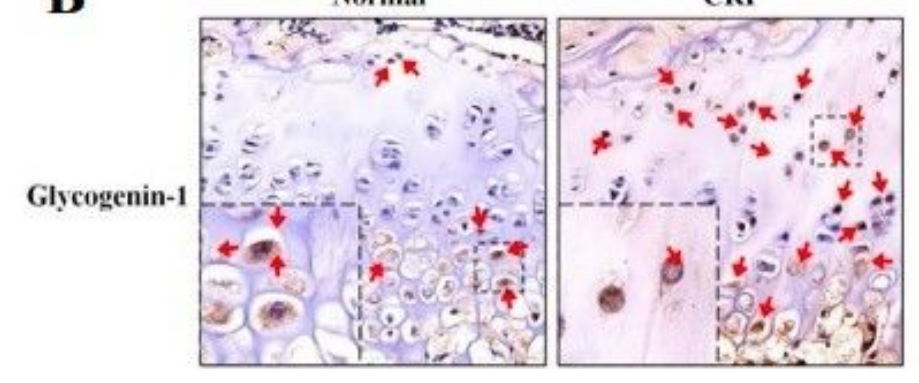

C

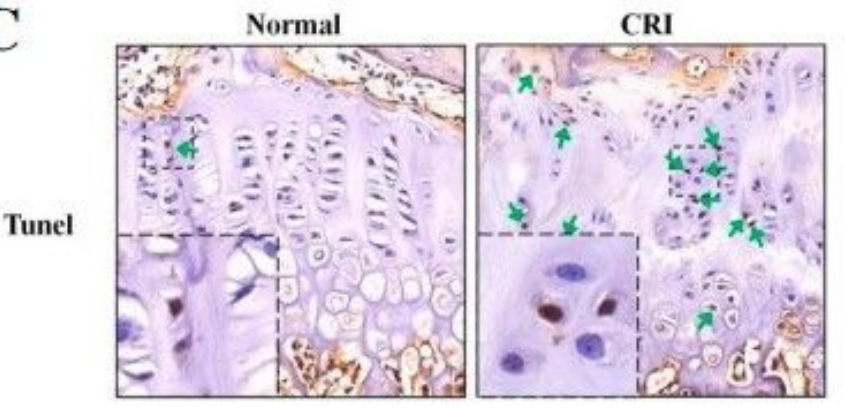

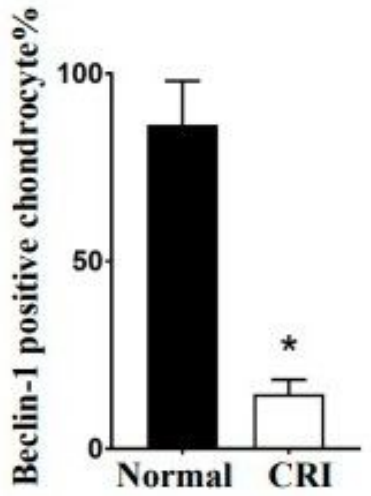
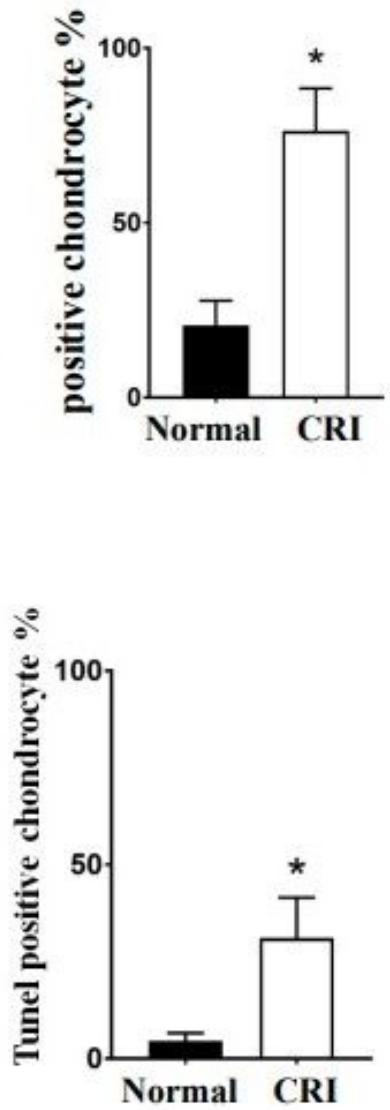

\section{Figure 3}

Immunohistochemical staining of histological sections of growth plates. (A) Immunofluorescence staining results

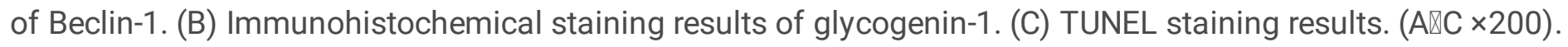
Bars represent the mean $\pm S E M, * p<0.05$ compared with the normal group. 
A
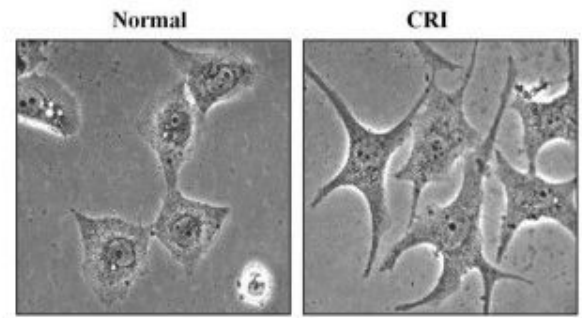

B
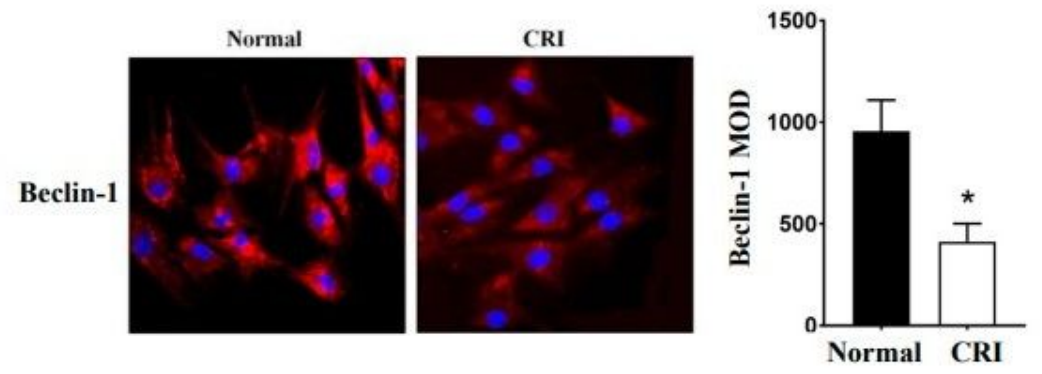

C
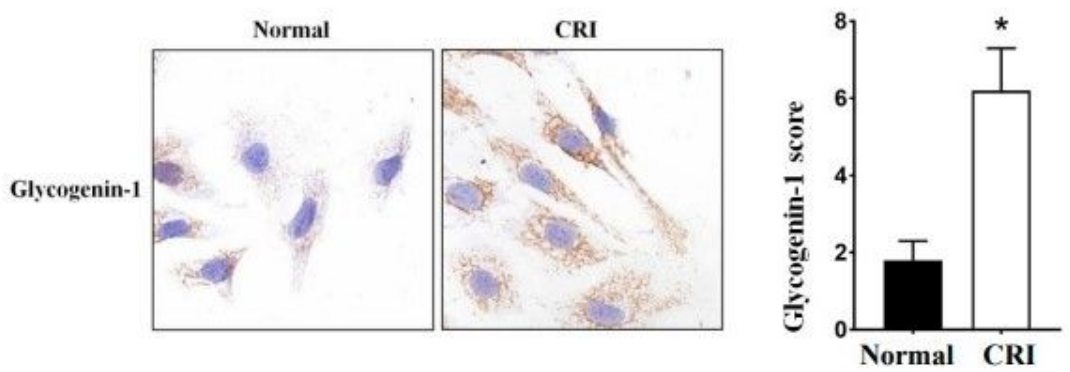

D

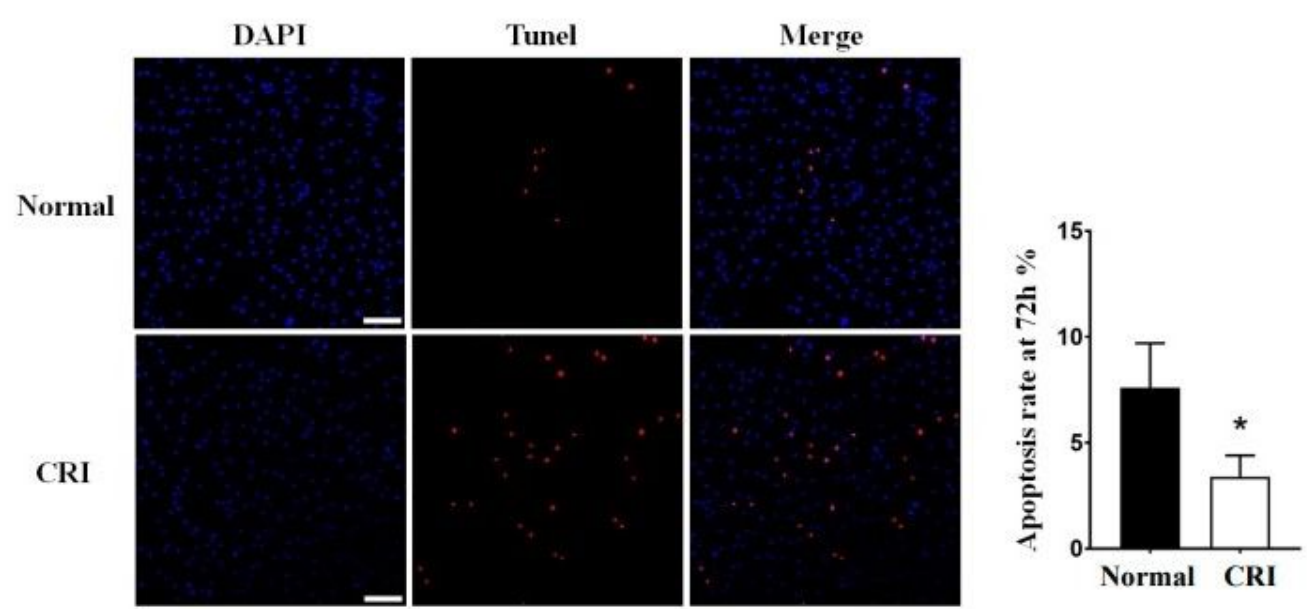

Figure 4

Immunohistochemical staining of growth plate chondrocytes in vitro. (A) Immunofluorescence staining results of Beclin-1 (×400). (B) Immunohistochemical staining results of glycogenin-1 (×400). (C) TUNEL staining results $(\times 100)$. Bars represent the mean $\pm S E M, * p<0.05$ compared with the normal group. 

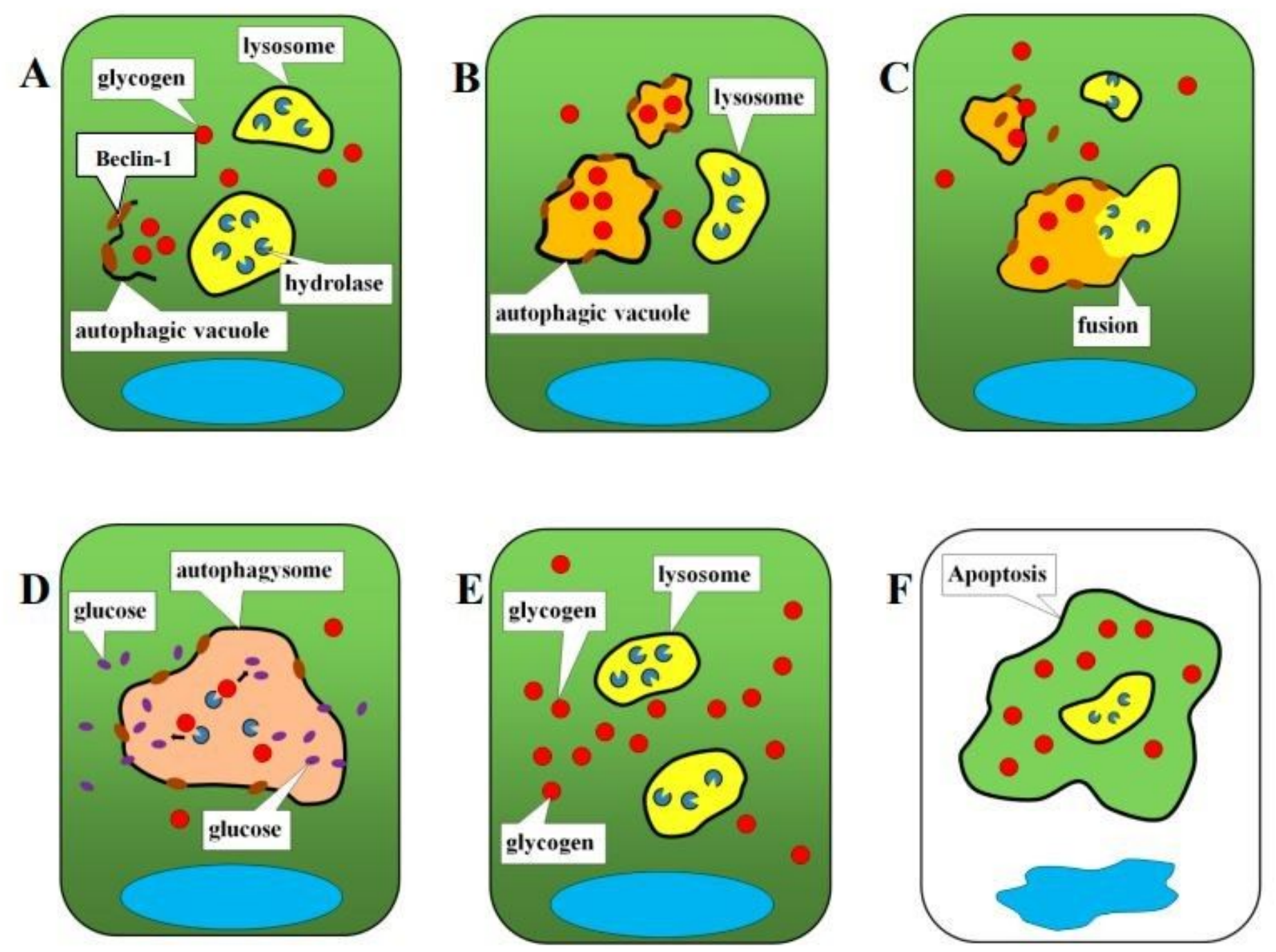

\section{Figure 5}

Proposed model for the role of chondrocyte autophagy in intracellular glycogen degradation. (A) Normal chondrocytes form autophagic vacuoles. (B) Normal chondrocyte autophagic vacuoles engulf glycogen particles. (C) Autophagic vacuoles fuse with lysosomes to form autolysosomes, and the hydrolases in the lysosome contact the glycogen particles. (D) The hydrolases in the autolysosome degrade the glycogen particles into glucose, which can be directly used by chondrocytes. (E) CRI chondrocytes cannot form autophagic vacuoles, and a substantial quantity of glycogen particles in the cytoplasm cannot be engulfed. (F) CRI chondrocytes do not have adequate glucose production in the cytoplasm for cellular functions, and chondrocyte apoptosis occurs due to insufficient energy. 